\title{
Educación a distancia y virtual: calidad, disrupción, aprendizajes adaptativo y móvil
}

\author{
Distance and virtual education: quality, disruption, \\ adaptive learning and mobile learning
}

\author{
Lorenzo García Aretio \\ UNED (España)
}

\section{Resumen}

Frente a las resistencias, la educación a distancia y digital va tomando ventaja sobre los formatos presenciales. Cuando los diseños pedagógicos son acertados, la calidad de los aprendizajes digitales está probada. La eficacia de estos sistemas es, al menos, similar a la de los presenciales. Por otra parte, se plantea en este trabajo el debate sobre las innovaciones y tecnologías disruptivas, proponiendo que el aprendizaje digital (nueva versión de la educación a distancia) está suponiendo una disrupción educativa porque plantea un cambio drástico de soportes y métodos y porque progresivamente va ganando espacio a los formatos convencionales. Y, finalmente, afirmamos que esta tendencia no está agotada, sino que continúa penetrando y ahondando la ruptura con las nuevas tecnologías, como, por ejemplo, las analíticas de aprendizaje, el aprendizaje adaptativo y el aprendizaje móvil.

Palabras clave: educación a distancia; aprendizaje digital; calidad y eficacia; educación disruptiva; analíticas de aprendizaje; aprendizaje adaptativo; aprendizaje móvil.

\begin{abstract}
Although faced with resistances, distance and digital education is overtaking face-to-face formats. When pedagogical designs are successful, there is evidence of the quality of digital learning. The effectiveness of these systems is at least similar to that of face-to-face proposals. This paper introduces the debate on innovations and disruptive technologies, arguing that digital learning (as a new version of distance education) is an educational disruption both because it brings about a drastic change of media and methods and because it is continually gaining ground from conventional formats. Finally, it is claimed that this trend is not exhausted, but continues to penetrate and deepen the break with new technologies, such as learning analytics, adaptive learning and mobile learning.
\end{abstract}

Keywords: distance education; digital learning; quality and effectiveness; disruptive education; learning analytics; adaptive learning; mobile learning.

Muy avanzada la segunda década del siglo XXI, nos seguimos enfrentando a numerosas resistencias a las innovaciones educativas que en el caso que nos va a 
ocupar, no suponen otra cosa que tratar de resistirse a la democratización del acceso a una educación de calidad sin restricciones de tiempo, espacio, ritmos de aprendizaje, vida laboral, ocupaciones familiares, libertad de movimiento, nivel educativo, etc. Generalmente, estas resistencias suelen provenir de determinadas administraciones públicas de algunos países y, también, de grupos más o menos influyentes que, por diferentes causas, se oponen a estos formatos educativos. Pero son resistencias generalmente pobres en argumentos y rigor. Muchas son las negativas para adoptar estos modelos educativos que, simplemente, se apoyan en altas dosis de ignorancia sobre la probada eficacia y calidad de estos modelos y de sus constatables prácticas exitosas en numerosos países e instituciones.

En 2009 publicábamos un libro que, muy conscientemente, titulábamos ¿Por qué va ganando la educación a distancia? (García Aretio, 2009). Con ese título, ya entonces, dábamos por supuesto que, en efecto, estamos convencidos de que, en cualesquiera de sus formulaciones, la modalidad, la metodología, las prácticas, las propuestas y los proyectos de educación a distancia han ido ganando terreno progresivamente y sin cesar, a lo que pudiéramos denominar como modalidad presencial o de docencia cara a cara sin mediación tecnológica. Esa afirmación nuestra, de esa u otra forma, ha sido asumida igualmente por otros autores, en algunos casos destacando iniciativas tales como los movimientos relativos a los Recursos Educativos Abiertos (REA, OER -Open Educational Resources-) o los propios MOOC (Massive Open Online Course) (Bayne, Knox y Ross, 2015).

Estas prácticas a distancia van progresivamente arrebatando espacio y tiempo a las formas más convencionales de enseñar y aprender, las metodologías a distancia que priman el trabajo autónomo de los estudiantes, así como las actividades cooperativas y colaborativas donde estos mismos participantes aprenden con otros, de otros y para otros, a través de las redes sociales, a través de comunidades de aprendizaje residentes en soportes digitales o, lo que en la última década ha venido siendo más habitual, a través de plataformas virtuales o entornos virtuales de aprendizaje diseñados con finalidades docentes. Así observamos con satisfacción que, ciertamente, esta modalidad va conquistando terreno. Cada vez hay un mayor número de instituciones, de programas, de docentes e investigadores comprometidos, más estudiantes que confían en esta modalidad y, lo que es más importante, los resultados de investigaciones sobre eficacia y eficiencia que se vienen mostrando, calidad, al fin y al cabo, aparecen al menos como de nivel similar a los de los procesos presenciales.

Un reciente trabajo (Allen y Seaman, 2016) muestra que a finales de 2014 en Estados Unidos existían 5,8 millones de estudiantes cursando estudios en línea y que entre 2013 y 2014, el crecimiento había estado en el 3,9\% (el año anterior, 3,7\%) y podríamos intuir que, hasta la actualidad, ese incremento no habrá sido menor. En el citado estudio se señala que el $28 \%$ de ese total de estudiantes toman al menos un curso en línea. 


\section{LA CALIDAD}

Respecto a la calidad, eficacia y eficiencia, algunos estudios realizados por nosotros mismos (García Aretio, 1985 y 1997) que tuvieron como población de análisis, por una parte, a los licenciados de la UNED y por la otra a los estudiantes y abandonos de esa misma universidad, llegan a concluir que la eficacia de un proceso educativo no está en la modalidad, sino en el rigor de los planteamientos pedagógicos que sustentan el diseño y desarrollo del proyecto.

Por otra parte, ya en 2010, tratamos de probar a través de una recopilación de investigaciones que han tenido por objeto contrastar resultados de aprendizaje en programas e instituciones de educación presencial frente a las de educación a distancia y virtual, que aquellos primeros análisis por nosotros realizados, tenían un sólido respaldo (García Aretio y Ruíz Corbella, 2010). En este estudio logramos recopilar revisiones, análisis y meta-análisis que vienen a agrupar en torno a unos 1000 trabajos, los cuales nos llevan a concluir que no existen diferencias significativas entre los resultados obtenidos en los formatos presenciales y en la modalidad a distancia y en línea. Si se desciende al detalle, podríamos apurar que la tendencia es favorable a los estudios a distancia. Los autores en los que nos basamos para afirmar lo que decimos, realizaron estudios fundados en numerosos trabajos científicos que se plantearon esta problemática.

Por época cercana a la de nuestro precedente estudio, una publicación del Departamento de Educación de Estados Unidos (Means, Toyama, Murphy, Bakia y Jones, 2009), realiza una búsqueda sistemática de la literatura de investigación desde 1996 hasta julio de 2008. En esa búsqueda se identificaron más de un millar de estudios empíricos de aprendizaje en línea. La selección de estos trabajos se basó en el contraste con la enseñanza presencial, en la medida de los resultados de aprendizaje. Pues bien, como promedio, los estudiantes en el aprendizaje en línea mostraron mejores resultados que los que recibieron programas cara a cara. Por otra parte, fueron superiores a ambas prácticas los resultados de aquellos estudiantes que siguieron la modalidad de blended-learning .

Un informe posterior, de otra revisión y meta-análisis sobre numerosas investigaciones relacionadas con este tema, continúa insistiendo en que no existen diferencias significativas entre los resultados obtenidos en procesos formativos presenciales o virtuales (Carey y Trick, 2013). Siemens, Gašević y Dawson (2015) publicaron otro informe de amplia difusión internacional que realiza una revisión de síntesis de investigación de destacadas evidencias y meta-análisis sobre la enseñanza a distancia, el blended-learning y la enseñanza digital, en el que llegan a concluir que existen grandes evidencias respecto a la efectividad de la educación a distancia, incluyendo algunos estudios que destacan que dicha efectividad es comparable e, incluso, superior a la de la enseñanza tradicional. Más recientemente, Allen y Seaman (2017), en el informe anual del estado de la educación en línea en los Estados Unidos, apuntan que el 35,6\% de los líderes académicos calificaron los resultados 
de aprendizaje en la educación mixta, blended o semipresencial como similares o superiores a los de la enseñanza cara a cara. El 63,3\% de esos líderes consideran a la educación en línea como fundamental para la estrategia institucional a largo plazo, frente al $13,7 \%$ que considera que no.

En realidad, las conclusiones avalan lo que ya viene siendo común en este tipo de estudios, es decir, que el rendimiento de los estudiantes depende más de los diseños pedagógicos de cada acción formativa que de los recursos seleccionados para el aprendizaje. Con diseños rigurosos, sean en formatos presenciales o en modalidad a distancia, los resultados no difieren significativamente. En consecuencia, el caso es que a estas alturas del siglo XXI se hace difícil poner en duda la eficacia y eficiencia de proyectos educativos no presenciales que estén soportados por sólidos diseños pedagógicos.

\section{¿POR QUÉ GANA ESTE SISTEMA?}

Ya hemos tratado de explicitar la calidad y eficacia probada de los sistemas educativos a distancia y virtuales, siempre que se actúe con criterios de rigor. Ahora, de forma resumida, trataremos de mostrar algunos de los factores que pueden estar impulsando el auge de estas modalidades formativas, más allá de la calidad probada ya apuntada. ¿Por qué la buena educación a distancia y virtual gana terreno, tanto desde la perspectiva social como desde la de quien elige el sistema para aprender? Existe numerosa literatura que incide en las ventajas y beneficios de la educación a distancia y virtual (Appana, 2008; Arkorful y Abaidoo, 2015; Bramble y Panda, 2008; Carey y Trick, 2013; Ekren y Kumtepe, 2016; García Aretio, 1994, 2001, 2009, 2010 y 2014; Guri-Rosenblit, 2009; Howard, Schenk y Discenza, 2004; Moore, 2013; Rodríguez, 2014; Sun y otros, 2008). Así, según estos autores, ¿̇cuáles pueden ser las causas de que avancen a gran ritmo las propuestas educativas a distancia?:

- Apertura. Porque desde la misma institución puede realizarse una amplia oferta de cursos; a los destinatarios no se les exige concentración geográfica y pueden encontrarse muy dispersos; la multiplicidad de entornos, niveles y estilos de aprendizaje puede mostrarse muy diferenciada; puede darse respuesta a la mayoría de las necesidades actuales de formación; puede convertirse en una oportunidad y oferta repetible sucesivamente.

- Flexibilidad. Porque permite seguir los estudios sin los rígidos requisitos de espacio (¿dónde estudiar?), asistencia y tiempo (¿cuándo estudiar?) y ritmo (¿a qué velocidad aprender?), propios de la formación tradicional; combinar familia, trabajo y estudio; permanecer en el entorno familiar y laboral mientras se aprende; compaginar el estudio también con otras alternativas de formación.

- Eficacia. Porque convierte al estudiante ineludiblemente en el centro del proceso de aprendizaje; puede aplicarse con inmediatez lo que se aprende; se facilita la integración de medios y recursos en el proceso de aprendizaje; se propicia la 
autoevaluación de los aprendizajes; los mejores especialistas pueden elaborar los materiales de estudio; la formación puede ligarse a la experiencia y al contacto inmediato con la actividad laboral que pretende mejorarse; los resultados referidos a logros de aprendizaje se muestran, al menos, de igual nivel que los adquiridos en entornos presenciales.

- Inclusión/democratización. Porque se abren oportunidades a segmentos sociales vulnerables y con dificultades habituales para acceder al bien de la educación; la bandera de la educación inclusiva bien que puede defenderse desde estos postulados; se supera el acceso limitado a la educación que provocan los problemas laborales, de residencia, familiares, etc.; se hace realidad la universalidad de la información; todos pueden acceder a todo tipo de documentos textuales y audiovisuales de los más prestigiosos autores.

- Economía. Porque se ahorran gastos de desplazamiento; se evita el abandono del puesto de trabajo; se disminuye el tiempo complementario de permanencia en el trabajo para acceder a la formación; se facilitan la edición y los cambios que se deseen introducir en los materiales; se propicia la economía de escala. Aunque estos presupuestos de índole económica están sometidos a revisión.

- Formación permanente. Porque se da respuesta a la gran demanda de formación existente en la sociedad actual; se muestra como ideal para la formación en servicio, la actualización y el reciclaje; se propicia la adquisición de nuevas actitudes, intereses y valores.

- Motivacióne iniciativa. Porque es inmensa la variedad y riqueza de la información disponible en Internet; permite la navegación libre por sus páginas; se presenta con un atractivo carácter multimedia; la libertad al navegar y la interactividad de las páginas Web mantienen la atención y propician el desarrollo de la iniciativa.

- Privacidad. Porque se favorece la posibilidad de estudiar en la intimidad; se evita lo que para muchos puede suponer la presión del grupo; se invita a manifestar conocimientos o habilidades que en presencia se obviarían; no se obliga a una exposición social, propia de la presencial, en personas que no la desean.

- Individualización. Porque se propicia el trabajo individual de los alumnos ya que cada uno puede buscar y consultar lo que le importe en función de sus experiencias, conocimientos previos e intereses; las tecnologías facilitan esa atención individualizada.

- Interactividad e interacción. Porque hace posible la comunicación total, bidireccional y multidireccional; la relación se convierte en próxima e inmediata; se posibilita la interactividad e interacción tanto síncrona como asíncrona, simétrica y asimétrica.

- Aprendizaje activo. Porque el estudiante es más sujeto activo de su aprendizaje; ese autoaprendizaje exige en mayor medida la actividad, el esfuerzo personal, el procesamiento y un alto grado de disciplina e implicación en el trabajo.

- Socialización. Porque se propicia el trabajo en grupo y el cultivo de actitudes sociales; se permite el aprender con otros, de otros y para otros a través del 
intercambio de ideas y tareas, y ello puede ser con personas muy distintas y distantes, lo que favorece la multiculturalidad.

- Autocontrol. Porque se fortalece la capacidad de autogestión del tiempo, del esfuerzo personal y de la conformación de itinerarios formativos; se potencia la capacidad de autodisciplina y de madurez.

- Macro-información. Porque pone a disposición del que aprende la mayor biblioteca jamás imaginada; ninguna biblioteca de aula, centro o universidad por sí misma alberga tantos saberes como los depositados en esta biblioteca cósmica.

- Gestión de la información. Porque propicia que el estudiante pase de mero receptor de información a convertirse en gestor y creador; incentiva la capacidad de buscar, valorar, seleccionar, recuperar inteligentemente la información, construir el conocimiento y llegar a publicarlo.

- Inmediatez. Porque la respuesta ante las más variadas cuestiones se ofrece a gran velocidad (a golpe de "clic"), al margen de la hora y el lugar.

- Innovación. Porque estimula formas diferentes y creativas de enseñar y aprender, tales como los aprendizajes combinados, colaborativos, invisibles, rizomáticos, ubicuos, adaptativos, etc.; el potencial de las comunidades que aprenden a través de las redes está modulando drásticamente los formatos habituales de educación.

- Permanencia. Porque la información no es fugaz como la de la clase presencial, la emisión de radio o televisión; el documento textual o audiovisual está esperando siempre el momento adecuado para el acceso de cada cual; quedan registrados todos los documentos e intervenciones como residentes en el sitio Web y ello posibilita las analíticas de aprendizaje.

- Multiformatos. Porque la diversidad de configuraciones que nos permiten las ediciones multimedia e hipertextual estimulan el interés por aprender; se brinda la posibilidad de ofrecer ángulos diferentes del concepto, idea o acontecimiento.

- Multidireccionalidad. Porque existe gran facilidad para que documentos, opiniones y respuestas tengan simultáneamente diferentes y múltiples destinatarios, seleccionados a golpe de "clic".

- Ubicuidad. Porque todos los participantes en el proceso de enseñanza y aprendizaje pueden estar virtualmente presentes en muchos lugares a la vez; la educación (el aprendizaje) ubicua y móvil agranda esta ventaja.

- Libertad de edición y difusión. Porque todos pueden editar sus trabajos y difundir sus ideas que, a la vez, pueden ser conocidos por multitud de internautas.

- Acceso a la calidad. Porque puede accederse a prestigiosos centros de estudio e investigadores sin necesidad de desplazamiento; los mejores pueden ser los autores de los materiales que se estudian.

- Interdisciplinariedad. Porque todos los ángulos, dimensiones y perspectivas de cualquier cuestión, problema, idea o concepto pueden ser contemplados desde diferentes áreas disciplinares y presentados de manera inmediata a través de los buscadores y enlaces hipertextuales. 


\section{LA DISRUPCIÓN}

En nuestro libro (García Aretio, 2014), apostábamos por considerar a la educación a distancia como un fenómeno disruptivo. Se habla de disrupción al referirnos a las tecnologías o innovaciones que son disruptivas cuando un producto o servicio nace y pasado un tiempo se convierte en líder sustituyendo a otro anterior (Christensen (1997 y 2012). Al menos, podríamos hablar de disrupción cuando ese producto o servicio sobrepasa en aceptación a los que estaban asentados en la sociedad y mercado. En estos casos se llega a generar un brusco cambio que rompe drásticamente y ocupa el espacio del producto o servicio anterior. Sólo por enumerar algunas tecnologías disruptivas en el sentido señalado, serían: el correo electrónico, la fotografía digital, la música en $\mathrm{CD}$, en $\mathrm{mp} 3$, por Internet, el teléfono móvil, los teléfonos inteligentes, la telefonía por IP, etc. Estas innovaciones llegaron a sustituir a las anteriores que venían ofreciendo servicios similares, pero de menor calidad y eficiencia que las nuevas. En algunos casos podrá discutirse si esas rupturas no son tales al tratarse de meras evoluciones.

Pues ese dilema es el que planteamos aquí. La incorporación o mejor, la integración de las tecnologías en los procesos educativos está obligando a cambios radicales en las estrategias de enseñanza y en los procesos de aprendizaje. ¿Podríamos hablar de innovaciones disruptivas? (Cabrol y Severín, 2010). ¿Es posible la innovación disruptiva en educación? (Christensen, Horn y Johnson, 2010). Estos autores abogan por esos cambios drásticos que han de producirse en la educación, algunos de ellos pregonados desde hace décadas en la literatura pedagógica pero que la práctica educativa no ha activado. Es ahora con la irrupción de tecnologías disruptivas en estas prácticas cuando toma valor ese cuestionamiento. De ahí que Adell y Castañeda (2012) apunten que para que haya una verdadera disrupción en las prácticas didácticas es necesario un cambio radical y repentino del contexto educativo, del marco conceptual didáctico y/o de los propios objetivos de la educación. Quiere decirse que por el solo hecho de impartir una asignatura o curso en línea, no se está llevando a cabo una innovación disruptiva. Probablemente no se esté alterando significativamente el proceso educativo por el mero cambio al soporte digital. Podríamos decir, de todas formas, que existe una cierta ruptura (disrupción) del clásico espacio donde se han venido desarrollando los procesos educativos ordinarios, el aula.

No sabemos hasta dónde podría considerarse así pero como ya hemos señalado, parecería que la educación a distancia en sus diferentes formatos digitales (aprendizaje digital), sobre todo en el nivel universitario y la formación profesional en servicio, en efecto, va ganando, va superando a los sistemas clásicos convencionales de educación y su presentación, al menos, supone un cambio radical del escenario educativo convencional. ¿Estaríamos entonces en condiciones de concluir en que la educación a distancia ha sido, está suponiendo, una disrupción pedagógica? ¿Quién 
podría dudar que, frente a los modelos pedagógicos clásicos, presenciales, existe ruptura? (García Aretio, 2015).

Es evidente que los modelos clásicos de educación no han desaparecido ni creemos que lo vayan a hacer en un plazo medio. Sin embargo, los niños, adolescentes y jóvenes ya no aprenden como lo hacían antes, sus formas de adquirir conocimiento están cambiando ¿̇adicalmente?, ¿No deberían hacer lo propio las formas de enseñar? ¿No resulta una alteración, un cambio, de primer orden, el aprendizaje que hoy se hace, también desde instituciones presenciales, a través de plataformas digitales (aprendizaje digital)?

Esas diferentes formas de enseñar y aprender soportadas en sistemas digitales van llegando a todos los rincones del planeta presentando formatos distintos según visiones pedagógicas, filosóficas, psicológicas, sociológicas y políticas y en función de las disponibilidades económicas y de los aportes tecnológicos. En efecto, como en otros ámbitos de la vida, son las tecnologías las que propician las disrupciones, también en la educación. En el caso que nos ocupa, los formatos educativos no presenciales se van imponiendo, en ocasiones, de forma brusca (disrupción), rompiendo espacios, tiempos, métodos, recursos, roles, etc., de sistemas educativos clásicos, estáticos, entumecidos, rígidos y con sólidas estructuras (García Aretio, 2014).

\section{EL FUTURO INMEDIATO}

Tras la imparable penetración de estas diferentes formas de enseñar y aprender que van rompiendo las maneras más tradicionales de hacerlo, consideraríamos si esta innovación educativa alcanzó ya su meseta de estabilidad, sigue profundizando la ruptura y sustitución de lo anterior o, por el contrario, ya está agotada. Para ello, nada mejor que acercarnos a los anuales informes Horizon. El correspondiente a este año (Horizon Report, 2017), como suele ser habitual, propone tendencias de base tecnológica en la innovación educativa, referido a la educación superior. Estos informes, año tras año, van consolidando ciertas tendencias, por una parte, aquellas que generan impacto, por otra parte, los retos a los que habremos de enfrentarnos y, finalmente, las tecnologías más relevantes que se adoptarán en el futuro. Ciertamente, las tecnologías aplicadas a la innovación educativa han tenido siempre una acogida especial y preferente en los entornos educativos no presenciales. De ahí que alimentemos nuestro convencimiento de que las metodologías a distancia, hoy digitales, lejos de detener sus avances, continuarán tomando terreno a los formatos educativos que durante siglos han configurado nuestros sistemas de formación.

En esta ocasión, con la finalidad de concretar estos avances, nos vamos a referir sólo a aquellas dos tendencias tecnológicas propuestas en el Horizon Report de 2017 de las que se marca su plena adopción en el plazo de un año. Dejaremos para otras ocasiones aquellas otras tecnologías cuya previsión de implantación serán a plazo mayor de un año. Pues bien, estas dos tecnologías referidas, son el aprendizaje 
adaptativo y el aprendizaje móvil, este último, tema de nuestro actual monográfico de RIED.

\section{Analíticas de aprendizaje y aprendizaje adaptativo}

Como en otras facetas de la vida, la parametrización de la acción y de los resultados pedagógicos con la finalidad de encontrar metodologías y procesos que lleven a aprendizajes deseados, ofrece interesantes perspectivas. Las analíticas de aprendizaje (learning analytics) derivan de otras analíticas de datos que se vienen utilizando en diferentes campos desde hace tiempo. El mundo del big data, los grandes datos, habitualmente utilizados en el ámbito empresarial para analizar las actividades del consumidor, identificar tendencias de consumo y predecir comportamientos, está siendo descubierto también para el sector educativo. Internet ha impulsado la investigación en torno a los big data y hacia métricas de todo tipo que explotan al máximo la información depositada en la Red por todos los internautas (Picciano, 2012). La minería de datos, el análisis de datos en grandes proporciones, ofrece a las empresas la posesión de inmensas bases de información que podrían utilizar, por ejemplo, en sus campañas de marketing.

El aprendizaje social a través de la red se está convirtiendo hoy en un campo de estudio muy complejo, debido fundamentalmente a los grandes avances tecnológicos y a la permanente demanda de formación por parte de la población, ello requiere de estudios y análisis serios que puedan propiciar formas de avanzar y mejorar la calidad de estas actividades de formación. De ahí la necesidad de analizar los datos, información y datos, por ejemplo, sobre el rastro que pueden dejar tras de sí los estudiantes durante un determinado proceso de formación en línea, su grado de interacción con docentes, compañeros y materiales de estudio, etc. Esto no es otra cosa que las analíticas de aprendizaje (Siemens et al., 2011; Buckingham y Ferguson, 2012), con las que tratamos de recopilar, medir, analizar y presentar multitud de datos sobre los estudiantes y sus contextos, con la finalidad de, primero entender y después tratar de optimizar los procesos de aprendizaje y el propio contexto en el que éste se produce (Long, Siemens, Conole y Gašević, 2011; Sclater y Mullan, 2017).

Ferguson (2012) señala los que considera factores o causas que propician los avances de las analíticas de aprendizaje en los entornos virtuales. Entre otros, apunta: el actual interés por los grandes datos, el auge de la formación virtual, las dudas sobre cómo saber el grado de implicación de un estudiante al que no vemos físicamente, la preocupación social por la rendición de cuentas y los deseos de mejorar la educación en línea a través de datos objetivos. Resulta sencillo evaluar el progreso académico de los estudiantes, predecir su rendimiento, detectar potenciales problemas, mejorar la experiencia de aprendizaje, aumentar la retención y también el grado de satisfacción de éstos. Sin duda, los estudiantes podrían comprobar que los diseños se ajustan mucho más a sus necesidades, intereses y limitaciones (Johnson et al., 2012 y 2013). 
Estaríamos hablando así del aprendizaje personalizado (Johnson et al., 2012) como uno de los desafíos importantes de esta década y que hoy viene evolucionando a la idea de aprendizaje adaptativo que supondría ordenar la acción docente y todos los recursos pedagógicos implicados hacia la satisfacción de las necesidades generadas por el proceso de aprendizaje de cada sujeto. Las tecnologías hoy permiten que los sistemas hipermedia se adapten a las características del usuario y no al revés. Las tecnologías adaptativas que pueden capturar datos individuales y grupales pueden aproximarnos a unas estrategias docentes más perfiladas hacia grupos y alumnos concretos. The Higher Education Commission (2016) del Reino Unido, que es un órgano formado por líderes educativos, empresariales y políticos, considera que el análisis de datos tiene el potencial de transformar el sector de la educación superior, aunque actualmente no aprovechan al máximo las oportunidades de las analíticas de aprendizaje. El informe precisa que el futuro puede mejorar impulsado por estos análisis basados en datos.

Las nuevas versiones de los LMS incorporan herramientas adaptativas que nos van permitiendo diseñar cursos individualizados o diferenciados (Leris, Vea y Velamazán, 2015), con prestaciones tales como gestionar el curso, a los usuarios, a los grupos, así como las calificaciones, etc., además de permitir, obviamente, la gestión de contenidos (García Peñalvo y Seoane-Pardo, 2015).

Poder controlar, por ejemplo, un curso virtual y adoptar decisiones y estrategias rápidas, no arbitrarias sino fundadas en análisis objetivos, supone un apoyo sustancial a docentes que no tienen por qué estar preparados pedagógicamente para implementar determinadas teorías o modelos para la mejora de los resultados académicos de sus estudiantes. Un informe de la empresa Tyton Partners (2016) estudia la evolución del aprendizaje adaptativo desde 2012. En ese informe se afirma que esta tecnología se utiliza para tratar una amplia gama de necesidades de aprendizaje, a partir de numerosas herramientas y aplicaciones específicas. Por su parte, los docentes parece que comienzan a fijarse en este tipo de análisis que podría propiciar mejores resultados de aprendizaje. Incluso las competencias se pueden evaluar con mayor claridad gracias a soluciones de aprendizaje adaptativo que apoyan formas claras de evaluación. Quizás falta que aumente el número de proveedores que ofrecen este tipo de productos. Sclater y Mullan (2017) creen que los sistemas de aprendizaje adaptativo ayudan a fomentar un acercamiento más personalizado y de ritmo propio al aprendizaje y son los más apropiados para enseñar habilidades básicas.

Cierto que no sería necesario analizar miles de registros para llegar a la conclusión de que existe correlación entre el esfuerzo de los estudiantes, la participación activa en el entorno virtual, la lectura de documentos, etc., y su rendimiento académico (Domínguez, Álvarez y Gil, 2016). Sin embargo, resultaría más difícil comprobar cuál sería la relación causal entre hacer, hacer más y hacerlo mejor (Reich, 2015).

Finalmente, una reflexión, habrá de actuarse con cautela con estas tecnologías, dados los componentes éticos y legales que pueden afectar a la explotación de ciertas 
bases de datos que contienen informaciones muy sensibles relacionadas con la privacidad de las personas.

\section{Aprendizaje móvil}

La ilusión de poder estar conectados siempre, sin importar tiempo ni espacio, ya no es una entelequia. La comunicación con todos y permanentemente, así como el acceso al inabarcable mundo de información que aloja Internet ya deja de estar limitado a un ordenador atado al espacio concreto por, al menos, dos cables, uno para la alimentación eléctrica y el otro para el acceso a Internet. Los dispositivos móviles, hoy fundamentalmente los teléfonos inteligentes, las tabletas y los relojes, permiten a los usuarios hacer esto realidad simplemente a través de las redes móviles e inalámbricas y de una cada vez mayor capacidad de autonomía energética. La curva referida al progresivo número de usuarios en todos los países del globo, unida a la evolución y suma de prestaciones que estos dispositivos ofrecen ha abierto la puerta a una gran cantidad de usos cotidianos. La omnipresencia de estos dispositivos está cambiando las maneras de interacción entre las personas, la información y el entorno (Informe Horizon, 2017).

De ahí que todo tipo de empresas y organizaciones se esfuercen por hacer asequibles sus sitios web desde estos dispositivos móviles realizando las adaptaciones de software precisas. Naturalmente, en el ámbito educativo esto se está convirtiendo en un gran reto tanto de las instituciones educativas como delas empresas generadoras de aplicaciones optimizadas para estos dispositivos móviles, porque, en efecto, la perspectiva más prometedora del aprendizaje móvil hoy son las aplicaciones, mundo que se ha convertido en un auténtico vivero de desarrollo que genera el consecuente negocio. En el mundo de la educación, el aprendizaje móvil conforma un grupo de espectaculares expectativas para este negocio del que, obviamente, podemos salir beneficiados todos los que nos dedicamos a este campo. A nivel mundial, a finales de 2012 se llegaba a un total de mil millones de teléfonos inteligentes, cantidad que se incrementará en un 34\% entre los años 2016 y 2022 (Sui y Wu, 2016). Por su parte, ITU (2016) afirma que el 95\% de las personas de los países desarrollados cuentan con un teléfono móvil y no es menos importante resaltar que en aquéllos que se encuentran en vías de desarrollo, lo poseen el 90\% de las personas. Otro interesante dato es el que aporta StatCounter (The Guardian, 2016) al informar que en 2016 el 51,3\% de la navegación web en todo el mundo se realizaba a través de teléfonos móviles y tabletas, superando por primera vez la navegación a través de dispositivos de escritorio.

La profusión de herramientas o aplicaciones móviles hacen que resulte extraño que no exista una aplicación para cada necesidad de aprendizaje (Johnson y otros, 2013). Así, el acceso a las fuentes del conocimiento, el aprendizaje, es posible en cualquier momento y desde cualquier lugar (ubicuidad) (Burbules, 2102) e, incluso, en movimiento. El aprendizaje móvil (m-learning) lo queremos referir al uso de 
dispositivos móviles o inalámbricos con finalidades que fomenten el aprendizaje (Ng y y Cumming, 2015; Saleh y Bhat, 2015).

Poder aprender sin las limitaciones de espacio y desde diferentes dispositivos se convierte en un fenómeno de grandes posibilidades dado que aumenta las ventajas propias del aprendizaje flexible al romper aún más las barreras espaciotemporales. El impacto sobre las posibilidades de aprender a través de dispositivos móviles en cualesquiera de los niveles educativos, resulta hoy incuestionable (Sung, Chang y Liu, 2016), y los elementos motivadores, de satisfacción e interés que muestran los estudiantes ante estas herramientas de aprendizaje parecen también muy positivos (Kim, Hwang y Zo, 2016), por las posibilidades de interacción y colaboración que se establecen entre los usuarios (Valk y otros, 2010; Sergio, 2012).

Para Santiago, Trabaldo, Kamijo y Fernández (2015), el m-learning muestra diferentes ventajas, tales como la transportabilidad, accesibilidad, conectividad permanente, flexibilidad, inmediatez y ubicuidad, características todas ellas que eliminan drásticamente las limitaciones espaciotemporales para el estudio, se realice éste de forma individual o colaborativa. La tecnología móvil facilita un aprendizaje interactivo, ubicuo e individualizado, que permite a cada usuario trabajar a su propio ritmo (Rico y Agudo, 2016). En definitiva, el aprendizaje móvil debería posibilitar no sólo el aprendizaje a distancia, semipresencial (blended learning) y ubicuo, sino también autónomo (Pareja, Calle y Pomposo, 2016).

El aprendizaje ubicuo lo definen Hooft y otros (2007) como aquél que se genera en un ambiente en el que los alumnos pueden acceder a diferentes dispositivos y servicios digitales, así como a los dispositivos móviles, siempre y cuando los necesiten. Puede observarse que estos autores consideran el aprendizaje ubicuo u-learning como un concepto más amplio que el m-learning, dado que incluye cualquier dispositivo con conexión a Internet. Es de tal calibre la penetración de Internet y de las redes sociales en la vida diaria que es ya bastante frecuente encontrar referencias y códigos (QR) en multitud de materiales y productos que hacen de puente para poder acceder a contenido extra en la web ya optimizada para visualizar en dispositivos móviles. Este es el caso de la realidad aumentada aplicada a la enseñanza (Ibáñéz, Jordano y Vermeulen, 2016).

La ubicuidad de estos formatos de aprendizaje rompe la dependencia y sujeción a un lugar concreto para llevar a cabo una sesión de aprendizaje. Así, el aprendizaje individual y colaborativo se hace realidad a través de estas tecnologías, con aplicaciones diferentes que responden a necesidades concretas de formación, con acceso a bases de datos, calendarios, chat, correo electrónico, videoconferencia, bibliotecas, acceso a redes sociales, blogs y wikis, además de posibilitar la conexión con el profesor y resto de estudiantes, consulta de contenidos en cualquier formato, etc. En realidad, todo lo que se puede hacer desde un ordenador de mesa conectado a Internet, con la ventaja de poderlo hacer desde cualquier lugar y tiempo y con dispositivos y aplicaciones más económicos. Sólo faltaría calcular los costes de las conexiones inalámbricas o wifi. A esta dificultad puede sumarse la del tamaño 
reducido de la pantalla y su brillo con luz solar, también la escasa capacidad de la memoria y duración de la batería, etc. En todo caso, tal y como señala Park (2011), urge un marco pedagógico que ajuste las metodologías más apropiadas de acuerdo con principios pedagógicos bien consolidados.

Quizás convendría concluir con una síntesis del documento de UNESCO (2013) que se dirige a responsables políticos, con la finalidad de orientar sobre qué es el aprendizaje móvil y cómo aprovecharlo para mejorar la educación. En el documento se recogen una serie de beneficios potenciales de estas tecnologías, tales como sus posibilidades para:

- Ampliar el alcance y la equidad de la educación.

- Facilitar el aprendizaje personalizado.

- Proporcionar feedback y evaluación inmediatos.

- Habilitar el aprendizaje en cualquier momento y en cualquier lugar.

- Asegurar un uso más productivo del tiempo que pasamos en clase (flipped classroom).

- Crear nuevas comunidades de aprendizaje.

- Apoyar el aprendizaje situado (realidad aumentada).

- Facilitar la continuidad de la experiencia de aprendizaje.

- Conectar el aprendizaje formal y el aprendizaje informal.

- Reducir la perturbación de la educación en casos de conflicto y desastres.

- Ayudar a los aprendices con discapacidades.

- Mejorar la comunicación y la administración.

- Minimizar los costes y maximizar la eficiencia.

En consecuencia, como venimos reforzando desde el inicio de este trabajo, la educación a distancia, el aprendizaje digital, no sólo está consolidado, no sólo está suponiendo una disrupción, sino que estos impulsos no van a decaer porque las tecnologías siempre supondrán un incentivo para la innovación educativa en ese campo. De esta manera, una apuesta en el sentido de potenciar estas tecnologías móviles para el aprendizaje la hace nuestra RIED. Revista Iberoamericana de Educación a Distancia, que ha promovido el presente monográfico relacionado con los dispositivos móviles y el aprendizaje, intentando siempre estar a la vanguardia de la educación digital.

\section{REFERENCIAS BIBLIOGRÁFICAS}

Adell, J., y Castañeda, L. (2012). Tecnologías emergentes, ¿̇pedagogías emergentes? En Tendencias emergentes en educación con TIC. Barcelona: Espiral.
Allen, E., y Seama, J. (2017). Digital Learning Compass: Distance Education Enrollment Report 2017. Babson Survey Research Group e-Literate, and WCET. 
Appana, S. (2008). A review of benefits and limitations of online learning in the context of the student, the instructor, and the tenured faculty. International Journal on ELearning, 7(1), 5.

Arkorful, V., y Abaidoo, N. (2015). The role of e-learning, advantages and disadvantages of its adoption in higher education. International Journal of Instructional Technology and Distance Learning, 12(1), 29-42.

Bayne, S.; Knox, J. y Ross, J. (2015). Open education: the need for a critical approach. Open education: the need for a critical approach, Learning, Media and Technology, 40:3, 247-250, doi: 10.1080/17439884.2015.1065272.

Bramble, W. J., y Panda, S. (Ed.). (2008). Economics of distance and online learning: Theory, practice and research. Londres: Routledge.

Buckingham, S., y Ferguson, R. (2012). Social Learning Analytics. Educational Technology and Society, 15(3), 3-26.

Burbules, N. (2012). Entrevista a Nicolás Burbulés. Webinar, 2012. Recuperado de http://goo.gl/DpJEcH.

Cabrol, M., y Severin, E. (2010). TICS en educación: una innovación disruptiva. Aportes BID, 2. Recuperado de: http:// www.iadb.org/wmsfiles/products/ publications/documents/35130690.pdf

Carey, T., y Trick, D. (2013). How Online Learning Affects Productivity, Cost and Quality in Higher Education: An Environmental Scan and Review of the Literature. Toronto: Higher Education Quality Council of Ontario.

Christensen, C. M. (1997). The innovator's dilemma: when new technologies cause great firms to fail. Boston: Harvard Business School Press.
Christensen, C. M., Horn, M. B. y Johnson, C. W. (2008). Disruptin class: How disruptive innovation will change the way the world learns. Nueva York: McGraw-Hill Professional.

Christensen, C. M. (2012). Disruptive innovation. En M. Soegaard y R. F. Dam (Ed.), Encyclopedia of human-computer interaction. Recuperado de: http://www. interaction-design.org/encyclopedia/ disruptive innovation.html .

Domínguez, D., Álvarez, J. F., y Gil-Jaurena, I. (2016). Analítica del aprendizaje y Big Data: heurísticas y marcos interpretativos. DILEMATA, International Journal of Applied Ethics, 22, 87-103. Recuperado de: $\quad$ http://www.dilemata.net/ revista/index.php/dilemata/article/ view/412000042/450

Ekren, G., y Kumtepe, E. G. (2016). Openness Initiatives in Distance Education. En Global Learn, 16(1), 346-351.

Ferguson, R. (2012). The State of Learning Analytics in 2012: A Review and Future Challenges. Technical Report. Milton Keynes: The Open University.

García Aretio, L. (1985). Licenciados extremeños de la UNED. Mérida: UNEDMérida.

García Aretio, L. (1994). Educación a distancia hoy. Madrid: UNED.

García Aretio, L. (Coord.) (1997). Investigar para mejorar la calidad de la Universidad. Madrid: UNED.

García Aretio, L. (2001). La educación a distancia. De la teoría a la práctica. Barcelona: Ariel.

García Aretio, L. (2009). ¿Por qué va ganando la educación a distancia? Madrid: UNED.

García Aretio, L. (2014). Bases, mediaciones y futuro de la educación a distancia en la sociedad digital. Madrid: Síntesis.

García Aretio, L., y Ruíz Corbella, M. (2010) La eficacia de la educación a distancia: 
¿un problema resuelto? Teoría de la educación, 22(1), 141-162.

García-Peñalvo, F. J., y Seoane-Pardo, A. M. (2015). Una revisión actualizada del concepto de eLearning. Décimo Aniversario. Education in the Knowledge Society (EKS), 16(1), 119-144, doi: http:// dx.doi.org/10.14201/eks2015161119144

Guri-Rosenblit, S. (2009). Distance education in the digital age: Common misconceptions and challenging tasks. Journal of Distance Education (Online), 23(2), 105.

Hooft, M., Swan, K., Cook, D., y Lin, Y. (2007). What is ubiquitous computing? En M. van Hooft y K. Swan (Eds.), Ubiquitous computing in education. Mahwah: Lawrence Erlbaum Associates.

Horizon Report (2017). The NMC Horizon Report: 2017 Higher Education Edition. Recuperado de https://goo.gl/3Udytu

Howard, C., Schenk, K., y Discenza, R. (Ed.). (2004). Distance learning and university effectiveness: Changing educational paradigms for online learning. IGI Global.

Ibáñez Moreno, A., Jordano de la Torre, M., y Vermeulen, A. (2015). Diseño y evaluación de VISP, una aplicación móvil para la práctica de la competencia oral. RIED. Revista Iberoamericana de Educación a Distancia, 19(1), 63-81. doi: http://dx.doi. org/10.5944/ried.19.1.14580

ITU-International Telecommunication Union. (2016). Measuring the Information Society Report. Recuperado de: $\quad$ http://www.itu.int/en/ITU-D/ Statistics/Documents/publications/ misr2016/MISR2016-w4.pdf

Johnson, L., Adams, S., y Cummins, M. (2012). The NMC Horizon Report: 2012 Higher Education. Austin, Tx: The New Media Consortium.
Johnson, L., Adams, S., Gago, D., García, E., y Martín, S. (2013). NMC Perspectivas Tecnológicas: Educación Superior en América Latina 2013-2018. Un Análisis Regional del Informe Horizon del NMC. Austin, Tx: The New Media Consortium.

Johnson, L., Adams, S., Cummins, M., y Estrada, V. (2012). Technology Outlook for STEM+ Education 2012-2017. NMC Horizon Report Sector Analysis. Austin, Tx: The New Media Consortium.

Kim. K., Hwang, J., y Zo, H. (2016): Understanding users' continuance intention toward smartphone augmented reality applications. Information Development, 32(2), 161-174.

Leris, D., Vea, F., y Velamazán, A. (2015). Aprendizaje adaptativo en Moodle: tres casos prácticos. Education in the Knowledge Society, 16(4), 138-157.

Long, P., Siemens, G., Console, G., \& Gasevic, D. (2011). 1st International Conference on Learning Analytics and Knowledge (LAK11), Banff, AB, Canada

Means, B., Toyama, Y., Murphy, R., Bakia, M., y Jones, K. (2009). Evaluation of Evidence-Based Practices in Online Learning: A Meta-Analysis and Review of Online Learning Studies. US Department of Education. Recuperado de https://eric. ed.gov/?id=ED505824

Moore, M. G. (Ed.). (2013). Handbook of distance education. Londres: Routledge.

Ng, W., y Cumming, T. M. (Eds.). (2015). Sustaining Mobile Learning: Theory, Research and Practice. Londres: Routledge.

Park, Y. (2011). A Pedagogical Framework for Mobile Learning: Categorizing Educational Applications of Mobile Technologies into Four Types. The International Review of Research in 
Open and Distance Learning, 12(2), 78102.

Picciano, A. G. (2012). The Evolution of Big Data and Learning Analytics in American Higher Education. Journal of Asynchronous Learning Networks, 16 (3), 9-20.

Reich, J. (2015). Rebooting MOOC Research. Improve assessment, data sharing, and experimental design. Science, 347(6217), 34-35. doi: 10.1126/science.1261627.

Saleh, S. A., y Bhat, S. A. (2015). Mobile Learning: A Systematic Review. International Journal of Computer Applications, 114(11), 1-5.

Santiago, R., Trabaldo, S., Kamijo, M., y Fernández, Á. (2015). Mobile Learning: Nuevas realidades en el aula. México: Editorial Océano.

Sclater, N., y Mullan, J. (2017). Learning Analytics in Higher Education: A Review of UK and International Practice. Recuperado de https://goo.gl/goroCB

Sergio, F. (2012). 10 ways that mobile learning will revolutionize education. Recuperado de http://www. fastcodesign.com/1669896/10-wayshat-mobilelearning-will-revolutionizeeducation

Siemens, G, Gasevic, D., Haythornthwaite, C., Dawson, S., Buckingham, S., Ferguson, R., Duval, E., Verbert, K., y Baker, R.S.J.D. (2011). Open Learning Analytics: an integrated \& modularized platform. Proposal to design, implement and evaluate an open platform to integrate heterogeneous learning analytics techniques. Recuperado de: https://goo. gl/ufr7zc

Siemens, G., Gašević, D., y Dawson, S. (Ed.), (2015). Preparing for the digital university: a review of the history and current state of distance, blended, and online learning. Edmonton, $\mathrm{AB}$ : Athabasca University.

Sui L., y Wu Y. (2016). Global Smartphone Sales Forecast for 88 Countries: 2007 to 2022. Strategy Analytics [blog]. Recuperado de: https://goo.gl/CsBYoi

Sung, Y. T., Chang, K. E., y Liu, T. C. (2016). The effects of integrating mobile devices with teaching and learning on students' learning performance: A meta-analysis and research synthesis. Computers \& Education, 94, 252- 275. doi: http:// dx.doi.org/10.1016/j.compedu.2015.11 .008

Sun, P. C., Tsai, R. J., Finger, G., Chen, Y. Y., y Yeh, D. (2008). What drives a successful e-Learning? An empirical investigation of the critical factors influencing learner satisfaction. Computers \& Education, 5O(4), 1183-12O2.

The Guardian (2016). Mobile web browsing overtakes desktop for the first time. Recuperado de https://goo.gl/SFLvb9

The Higher Education Commission (2016). From Bricks to Clicks - The Potential of Data and Analytics in Higher Education. Recuperado de https://goo.gl/s132y7

Tyton Partners (2016). Learning to Adapt 2.o: The Evolution of Adaptive Learning in Higher Education. Recuperado de https://goo.gl/nXOZZB

UNESCO (2013). UNESCO policy guidelines for mobile learning. París: UNESCO.

Valk, J. H., Rashid, A. T., y Elder, L. (2010). Using mobile phones to improve educational outcomes: An analysis of evidence from Asia. The International Review of Research in Open and Distance Learning, 11(1), 117-140. 


\section{Como citar este artículo:}

García Aretio, L. (2017). Educación a distancia y virtual: calidad, disrupción, aprendizajes adaptativo y móvil. RIED. Revista Iberoamericana de Educación a Distancia, 2o(2), pp. 09-25. doi: http://dx.doi.org/10.5944/ried.20.2.18737 